\title{
Political Violence and Child Height: Evidence from the 2003
}

\section{Casablanca Bombings}

\begin{abstract}
This research examines how in-utero exposure to political violence affects early childhood health within the context of the 2003 Casablanca Bombings in Morocco. Exploiting the variation across districts and birth months-years within a difference-in-differences framework, we uncover the detrimental association between in-utero exposure to the bombings and child height. Prenatally exposed children are 0.743 standard deviations shorter for their age. Children that were prenatally exposed to the bombings are 0.743 standard deviations shorter for their age. When examining the relative importance of exposure timing, we find that being exposed to the bombings during the first trimester had the most impact on a child's height.
\end{abstract}

